\title{
Repeated stimulation of cultured networks of rat cortical neurons induces parallel memory traces
}

\author{
Joost le Feber, ${ }^{1,2}$ Tim Witteveen, ${ }^{1}$ Tamar M. van Veenendaal, ${ }^{1,3}$ and Jelle Dijkstra ${ }^{1}$ \\ ${ }^{1}$ Department of Biomedical Signals and Systems, University of Twente, MIRA Institute for Biomedical Engineering and Technical \\ Medicine, Enschede 7500 AE, The Netherlands; ${ }^{2}$ Department of Clinical Neurophysiology, University of Twente, Enschede 7500 AE, \\ The Netherlands; ${ }^{3}$ Department of Radiology, Maastricht University, School for Mental Health and Neuroscience, Maastricht University \\ Medical Centre, Maastricht 6200 MD, The Netherlands
}

\begin{abstract}
During systems consolidation, memories are spontaneously replayed favoring information transfer from hippocampus to neocortex. However, at present no empirically supported mechanism to accomplish a transfer of memory from hippocampal to extra-hippocampal sites has been offered. We used cultured neuronal networks on multielectrode arrays and small-scale computational models to study the effect of memory replay on the formation of memory traces. We show that input-deprived networks develop an activity $\Leftrightarrow$ connectivity balance where dominant activity patterns support current connectivity. Electrical stimulation at one electrode disturbs this balance and induces connectivity changes. Intrinsic forces in recurrent networks lead to a new equilibrium with activity patterns that include the stimulus response. The new connectivity is no longer disrupted by this stimulus, indicating that networks memorize it. A different stimulus again induces connectivity changes upon first application but not subsequently, demonstrating the formation of a second memory trace. Returning to the first stimulus does not affect connectivity, indicating parallel storage of both traces. A computer model robustly reproduced experimental results, suggesting that spike-timing-dependent plasticity and short time depression suffice to store parallel memory traces, even in networks without particular circuitry constraints.
\end{abstract}

In vivo, consolidation of declarative memory can be subdivided into two specific processes (Squire et al. 1984). Rapidly after learning, memories are temporarily stored in hippocampus, a process generally referred to as synaptic consolidation. During the second phase, systems consolidation, memories are slowly transferred to the neocortex. This stage of memory encoding probably requires repeated activation of cortical areas by the hippocampus (Sutherland and McNaughton 2000; Frankland and Bontempi 2005; Karlsson and Frank 2009; Nakashiba et al. 2009). Longterm potentiation is generally assumed to be the underlying process in synaptic consolidation (Bramham and Messaoudi 2005). However, whether and to what extent the signs of reactivation play a functional role in consolidating respective neural memory representations or merely reflect use-dependent phenomena of inert neural activity is presently not clear (Gais and Born 2004). At present, no empirically supported mechanism to accomplish a transfer of memory from hippocampal to extra-hippocampal sites has been offered (Nadel et al. 2007) and the "algorithms" that create memory traces remain unclear, forming a major obstacle in the search for memory traces.

Studies of artificial neural networks suggested that the stabilization of reverberating neural activity underlying shortterm memory produces long-term memory (Gerard 1949; Hebb 1949). Later basic theories proposed that activity patterns in artificial recurrent excitatory networks are dictated by attractors, local minima in the energy landscape that are associated with certain activation patterns. External input substantially changes the set of attractors of a network (Amit 1989), and thus the palette of activation patterns, which may reflect memory traces. However, these theories cannot be straightforwardly validated in biological networks, because simultaneous activity of multiple neurons is

Corresponding author: j.lefeber@utwente.nl

Article is online at http://www.learnmem.org/cgi/doi/10.1101/lm.039362.115. difficult to record in vivo and consequently, it is hard to provide accurate estimates of the synaptic coupling in vivo.

Dissociated cortical neurons cultured on multielectrode arrays provide a useful platform to study network aspects of neuronal tissue, including memory. A week after seeding cultures become spontaneously active, to reach a mature state after $\sim 3 \mathrm{wk}$ (van Pelt et al. 2004; Stegenga et al. 2008). Beyond 3 wk, activity patterns and connectivity stabilize, but a slow drift of observed activity patterns (on timescales of hours to days) remains (Stegenga et al. 2008). Activity patterns are determined by a certain connectivity, and conversely, activity patterns affect connectivity through plasticity mechanisms like spike-timing-dependent plasticity (STDP). Networks develop an activity-connectivity balance, where activity patterns support current connectivity, connectivity appears relatively constant, and activity may fluctuate within a fixed set of possible patterns. Responses to electrical stimulation usually differ from spontaneously occurring patterns and therefore disturb the activity-connectivity balance, yielding a change in connectivity.

We hypothesize that networks will develop a new activityconnectivity equilibrium, such that the new spontaneous activity patterns include the network response to the applied stimulus. If so, the first application of a certain stimulus should activate new patterns, and induce connectivity changes. Repeated application of that same stimulus should not lead to further connectivity changes as the stimulus response becomes part of the spontaneous repertoire, and imposes no further drive away from the equilibrium. This reasoning provides a tool to verify the

\footnotetext{
C 2015 le Feber et al. This article is distributed exclusively by Cold Spring Harbor Laboratory Press for the first 12 months after the full-issue publication date (see http://learnmem.cshlp.org/site/misc/terms.xhtml). After 12 months, it is available under a Creative Commons License (AttributionNonCommercial 4.0 International), as described at http://creativecommons. org/licenses/by-nc/4.0/.
} 
hypothesis without a priori knowledge of the actual encoding of memory traces.

\section{Results}

We investigated the effect of repeated stimuli on connectivity in 21 experiments in 20 cultured cortical networks on multielectrode arrays, and in 36 simulations with a computer model of a 100-neuron cortical network.

\section{Experiments}

In all experiments, long-term recordings were subdivided into data blocks. We determined the connectivity Matrix S for each data block (see Materials and Methods) to assess connectivity changes. Without external input, functional connectivity was quite stable: on average, strengths of functional connections varied $<25 \%$ of their mean value for the duration of our experiments (5-10 h). In stimulation experiments, all evaluation periods between the stimulation periods contained multiple data blocks, each having their own connectivity $S$, with a certain distance to the initial connectivity. We calculated the mean distance to the initial connectivity, $\mathrm{ED}_{0}$, for all evaluation periods. Figure $1 \mathrm{~A}$ shows the temporal evolution of $\mathrm{ED}_{0}$ in control cultures $(n=4$, dashed line) and cultures that received $15 \mathrm{~min}$ of tetanic stimulation (see Materials and Methods) after every hour of no stimulation $(n=13$, solid line). There is no significant difference between the starting points of both curves ( $t$-test $P>0.38$ ). One-way ANOVA showed that $\mathrm{ED}_{0}$ significantly increased in stimulated cultures $(P<0.03)$, whereas control cultures showed no significant change $(P>0.4)$.

Low-frequency stimulation $(0.2 \mathrm{~Hz}$, during $15 \mathrm{~min})$ yielded comparable results, $\mathrm{ED}_{0 \text {,norm }}$ increased significantly in stimulated cultures (ANOVA, $P<0.003$ ). The distance between the connectivity before and after the first period of stimulation $\left(\mathrm{ED}_{\text {stim }}[1]\right)$ was much larger than $\mathrm{ED}_{\text {stim }}$ around subsequent stimulation periods (Fig. 1B, solid line). After normalization, all distances except $\mathrm{ED}_{\text {stim }}[2]$ were significantly smaller than $\mathrm{ED}_{\text {stim }}[1]$ ( $t$-test: $P<$ 0.03). The distance across stimulation periods did not drop to zero, but as shown in Figure 1B, the remaining connectivity differences did not yield an increasing distance from the initial connectivity $\left(\mathrm{ED}_{0}\right.$, dashed line). Figure $1 \mathrm{C}$ shows the mean area under the post-stimulus time histogram (PSTH) upon each stimulus pulse. The mean amount of action potentials in response to stimulation did not change during the experiment $(P=0.97$, one-way ANOVA). Finally, we estimated similarity between events (stimulus responses or spontaneous network bursts recorded before or after the stimulation period) using the EC measure (see Materials and Methods). Stimulus responses tended to have higher similarity to post-stimulation spontaneous bursts than to prestimulation burst, but the difference was not significant.

In seven cultures, we investigated the effect of stimulation at a second electrode. Both electrodes were selected to yield clearly detectable network responses (see Materials and Methods), without further restrictions related to their location. The distance between the stimulation electrodes ranged from $200 \mu \mathrm{m}$ (adjacent electrodes) to $1.34 \mathrm{~mm}$ (see Table 1). Results obtained with adjacent stimulation electrodes were similar to those obtained with larger distances between the stimulation electrodes, but we did not specifically search for possible distance dependencies. Similarity between responses to stimulation at one electrode, as assessed by event correlation (EC, see Materials and Methods), averaged 0.61, and was much higher than EC between the first stimulations at different electrodes. Figure 2A,B shows an example of
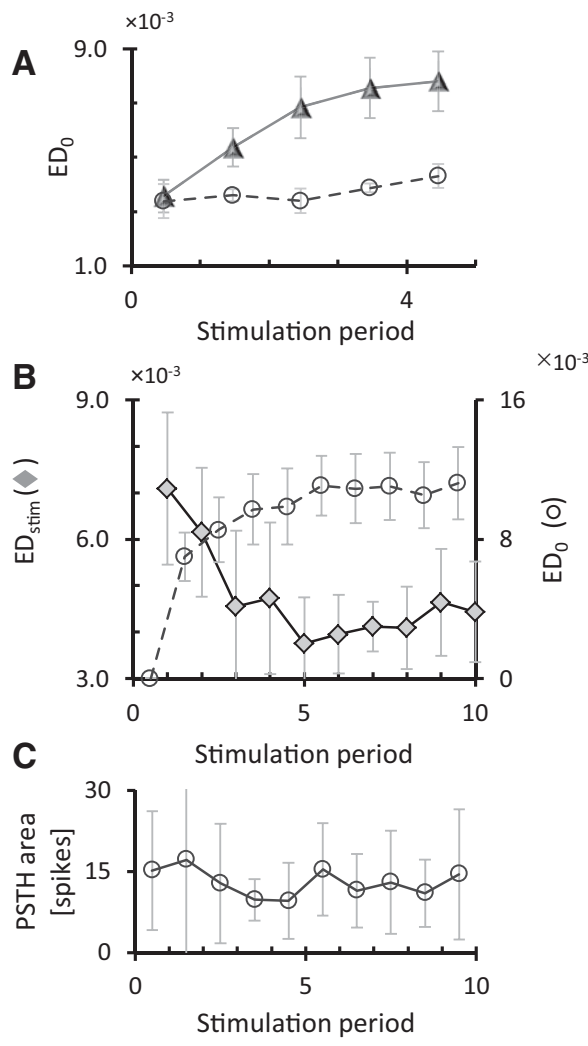

Figure 1. Connectivity changes in control recordings and repeatedly stimulated cultures. Long-term recordings were divided into data blocks, in each data block we calculated the connectivity matrix and we calculated the Euclidean distance from the initial connectivity matrix $\left(E D_{0}\right)$. $(A)$ Connectivity changes in control recordings $(O$, dashed line; $n=4)$ and repeatedly (tetanus) stimulated cultures ( $\Delta$, solid line; $n=$ 13). Horizontal axis indicates stimulation periods (15 min of tetanic stimulation), the intervals in between denote $1 \mathrm{~h}$ of spontaneous activity. The average distance in the first evaluation period reflects spontaneous fluctuations. Error bars indicate SEM and reflect differences between experiments. (B) Connectivity changes during repeated low-frequency stimulation $(0.2 \mathrm{~Hz}$ for $15 \mathrm{~min}, n=4)$. From the periods of spontaneous activity ( $1 \mathrm{~h}$ each) between stimulation periods, we calculated connectivity matrices and the distances from the initial connectivity (dashed line, $\bigcirc$ ) and from the connectivity before the last stimulation period (solid line, $\bullet$ ). Error bars indicate SEM and reflect differences between experiments. (C) Average area under the PSTH of all stimuli within each stimulation period. On average there were no significant changes in the number of stimulus induced action potentials throughout the experiments (ANOVA $P=$ 0.97 ). Total time along horizontal axis in $B$ and $C$ was $\sim 12 \mathrm{~h}$.

two responses to stimulation at different electrodes $\mathrm{A}$ and $\mathrm{B}$, with $\mathrm{EC}(\mathrm{A}[1], \mathrm{B}[1])=0.32$.

Periods of spontaneous activity were divided into $16.7 \pm 1.4$ data blocks to determine the temporal development of functional connectivity (see Materials and Methods). In all experiments, connectivity changes across stimulation periods leveled off when the first electrode was stimulated during four stimulation periods.

Subsequent switch to another electrode again yielded large connectivity changes upon the first stimulation followed by smaller changes after successive stimuli at that electrode. Finally, returning to the first stimulation electrode did not induce connectivity changes larger than spontaneous fluctuations. Figure 2C shows connectivity changes in response to stimulation at electrode $\mathrm{A}$ or $\mathrm{B}$, with respect to the connectivity just before the first application of that stimulus (i.e., switching to a new 
Table 1. Overview of cultures used

\begin{tabular}{|c|c|c|c|c|}
\hline Culture & DIV & Stim type & Electrode(s) & $\begin{array}{l}\text { Stimulation } \\
\text { electrode }\end{array}$ \\
\hline 1 & 33 & Tetanus & $A(4 x)$ & \\
\hline 2 & 15 & Tetanus & $A(4 x)$ & \\
\hline 3 & 33 & Tetanus & $A(4 x)$ & \\
\hline 4 & 20 & Tetanus & $A(4 x)$ & \\
\hline 5 & 14 & Tetanus & $A(4 x)$ & \\
\hline 6 & 30 & Tetanus & $A(4 x)$ & \\
\hline 7 & 18 & Tetanus & $A(4 x), B(4 x), A(4 x)$ & $A=17, B=77$ \\
\hline 7 & 20 & Tetanus & $A(4 x), B(4 x), A(4 x)$ & $\mathrm{A}=57, \mathrm{~B}=48$ \\
\hline 8 & 21 & Tetanus & $A(4 x), B(4 x), A(4 x)$ & $A=17, B=37$ \\
\hline 9 & 25 & Tetanus & $A(4 x), B(4 x), A(4 x)$ & $\mathrm{A}=74, \mathrm{~B}=73$ \\
\hline 10 & 19 & Tetanus & $A(4 x), B(4 x), A(4 x)$ & $A=53, B=78$ \\
\hline 11 & 19 & Tetanus & $A(4 x), B(4 x), A(4 x)$ & $\mathrm{A}=62, \mathrm{~B}=32$ \\
\hline 12 & 20 & Tetanus & $A(4 x), B(4 x), A(4 x)$ & $A=12, B=75$ \\
\hline 13 & 21 & Low frequency & $A(10 x)$ & \\
\hline 14 & 47 & Low frequency & $A(10 x)$ & \\
\hline 15 & 53 & Low frequency & $A(10 x)$ & \\
\hline 16 & 18 & Low frequency & $A(10 x)$ & \\
\hline 17 & 40 & None & & \\
\hline 18 & 29 & None & & \\
\hline 19 & 35 & None & & \\
\hline 20 & 36 & None & & \\
\hline
\end{tabular}

(DIV) days in vitro. Stimuli consisted of biphasic current pulses (12-24 $\mu \mathrm{A}$ $200-\mu$ sec per phase), which were delivered during stimulation periods of 10-15 $\mathrm{min}$ as low-frequency pulses (inter-pulse interval: $3-5 \mathrm{sec}$ ), or as tetani (trains of 10 pulses at $100 \mathrm{~Hz}$, inter-train interval: $5 \mathrm{sec}$ ). Cultures were stimulated either at a single electrode (A) or at two different electrodes (A and $B)$. The numbers between brackets indicate the number of stimulation periods. Electrode numbers refer to column and row; e.g., electrode 32 is the second electrode of the third column of the MEA.

stimulation electrode was associated with a new reference for $\mathrm{ED}_{0}$ ). Differences across first stimulation periods at either electrode were substantially larger than those across subsequent stimulation periods, or periods of equal duration of no stimulation. Returning to stimulus A, we found no connectivity changes larger than spontaneous fluctuations. On average, the array wide spontaneous activity remained constant during the experiments (ANOVA: $P>0.99$, see Fig. 2D).

\section{Computer modeling}

A model of 100 neurons coupled by synapses with short-term facilitation (STF) and depression (STD) and STDP, robustly reproduced the in vitro finding that networks develop an activity-connectivity balance. We used several network realizations, two different implementations of STDP, various patterns of synaptic noise, and low-frequency or tetanic stimulation. With additive STDP, the percentage of extreme synaptic weights $(>90 \%$ of their maximum or $<10 \%$ ) increased during the whole simulation and averaged $73 \%$ at the end of the simulation. With tetanic stimulation, the fraction of synapses with an extreme value decreased during all stimulation periods in all simulations, although in some periods this decline was small. Whereas low-frequency stimulation and additive
STDP often led to many synapses with extreme values, the combination of the multiplicative STDP model and tetanic stimulation generally yielded unimodally distributed synaptic strengths.

Activity and connectivity robustly stabilized in all networks explicitly tested $(n=5)$. To quantify the stability of connectivity, we calculated the temporal development of $\mathrm{ED}_{0}$. Connectivity stabilized as the slope of the $\mathrm{ED}_{0}$ curve against time approached zero (see Materials and Methods). The slope dropped below $0.5 \%$ within $\sim 30 \mathrm{~min}$. In all other models connectivity also stabilized, before we applied stimulation.

The models also reproduced the experimental finding that a first external stimulus induced large connectivity changes, reflected by large $\mathrm{ED}_{\text {stim }}$ values. Connectivity changes in response to low-frequency stimulation were substantially larger than those in response to tetanic stimulation. Although the differences were much smaller, we saw a similar trend in the experimental data. Subsequent stimuli did not induce large connectivity changes (tetanic stimulation), or to a rapidly decreasing extent (lowfrequency stimulation), as illustrated in Figure 3A.

In 12 simulations, we stimulated the network model for $2 \mathrm{~h}$ at $0.125 \mathrm{~Hz}$. To investigate the similarity between stimulus responses and spontaneous pre- and post-stimulation burst patterns, we calculated the events correlations EC (see Materials and Methods). The example in Figure 3B shows that stimulus responses progressively resembled their neighbors, indicating a stabilization of the response. Furthermore, the average similarity of stimulus responses to post-stimulation spontaneous bursts was significantly higher than that to prestimulation bursts (one-sided Wilcoxon signed rank, $P<0.001$ ).

A typical example of the responses to two different stimuli A and B (Fig. 4A) shows that the main difference occurred in the first part of the response. The later part of the response was generally relatively similar for different stimuli. Overall similarity of the response to these two stimuli was 0.95 , which would be indicated as green in Figure 3B. Provided that synaptic strengths did not reach
A

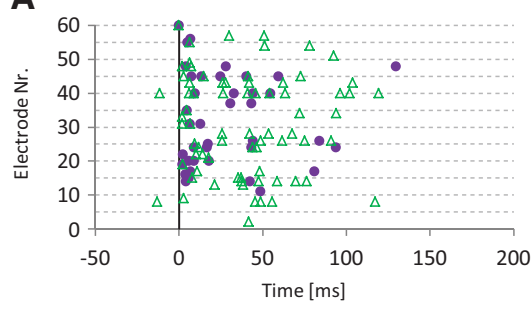

B

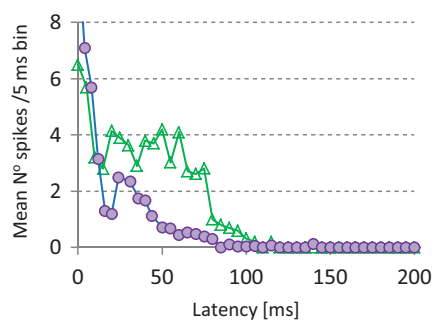

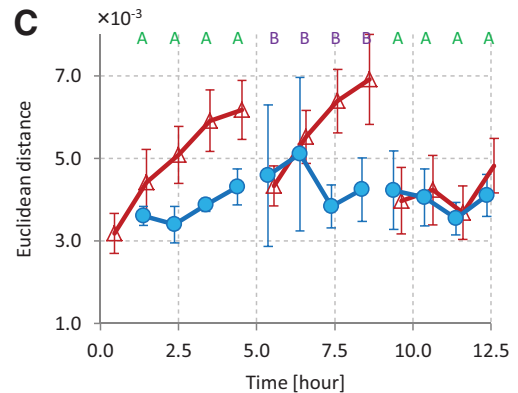

D

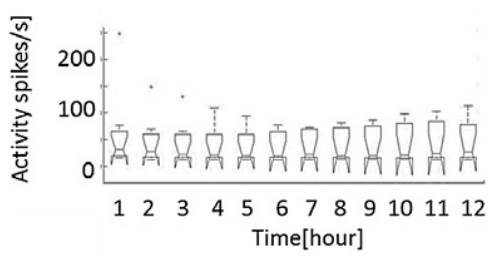

Figure 2. Network response to two different stimuli. (A) Example raster plots of responses to stimulus $A(\Delta)$ and $B(\bullet) \mathrm{EC}=0.32$. (B) Mean stimulus response to both stimuli $\left(n_{\mathrm{A}}=20, n_{\mathrm{B}}=20\right)$ in that experiment. (C) Euclidean distance from the initial connectivity matrix without stimulation $(\bullet, n=4$ experiments), or across periods of tetanic stimulation at electrode $A$ or $B$, as indicated $(\Delta, n=7$ experiments). For comparison, all distances are calculated to the last connectivity matrix before stimulation at that particular electrode $(A$ or $B)$. (D) Box plot of the spontaneous array wide firing rate. On each box, the central mark is the median, the edges of the box are the 25 th and 75 th percentiles, the whiskers extend to the most extreme data points considered to be not outliers, and the outliers are plotted individually. The firing rate remained unchanged during these experiments (one-way ANOVA, $P>0.99$ ). 
A

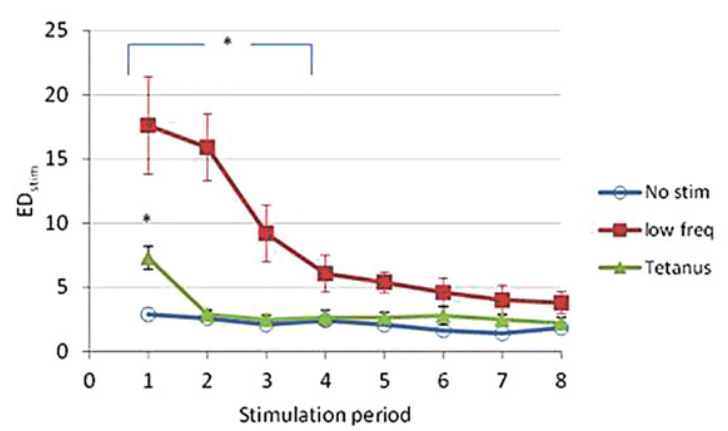

B

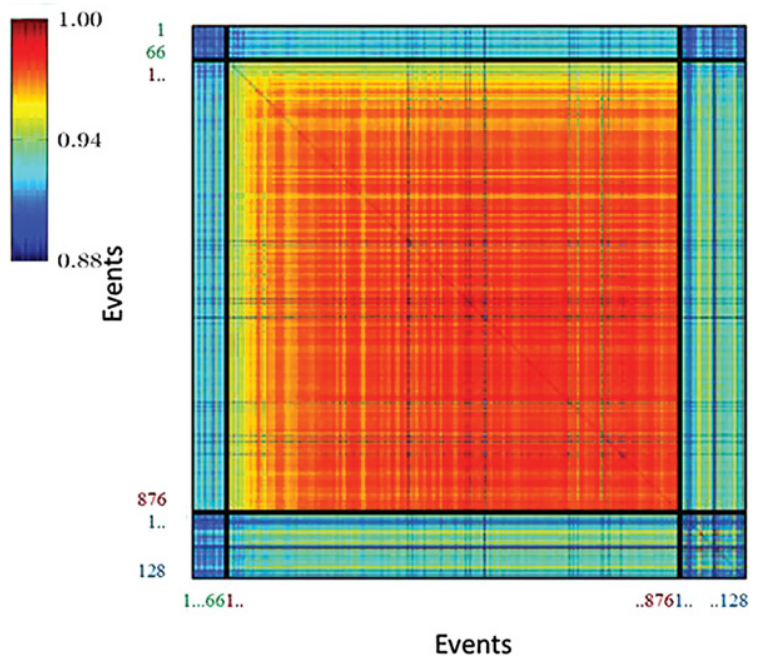

Figure 3. Connectivity changes following repeated stimulation in the computer model. (A) Squares show the effect of low-frequency stimulation $(0.2 \mathrm{~Hz}$ for $15 \mathrm{~min})$, triangles show tetanic stimulation. Both curves indicate connectivity changes across stimulation periods, averages \pm SDs of four simulations are shown. No stimulation (circles) was simulated once. Significant differences (ANOVA and Tukey HSD test, $P<0.01$ ) are indicated by an asterisk. (B) Typical example of the similarity between all events in a simulation: 66 prestimulation bursts (labeled in green), 876 stimulus responses (labeled in red), and 128 post-stimulation bursts (labeled in blue). The figure shows color-coded values of all EC $(n, m)$, $0<n, m \leq(66+876+128)$. Twenty-four of the 900 stimulations did not evoke a network response.

extreme values after application of the first stimulus (which in general was the case when tetanic stimulation was applied), a second, different stimulus also induced large connectivity changes at first, but not after subsequent stimulation periods. Return to the first stimulus did not induce $\mathrm{ED}_{\text {stim }}$ values that significantly exceeded spontaneous fluctuations. Figure 4 also displays $\mathrm{ED}_{0}$ (solid line, open circles) for easy comparison with Figure 2C.

\section{Discussion}

Spontaneous neuronal activity patterns reflect connectivity and closely timed spikes in coupled neurons may potentiate or depress the efficacy of transmission in the connecting synapse. Without external input, dissociated cortical networks develop rather stable firing patterns (Stegenga et al. 2008), as well as a stable connectivity (le Feber et al. 2007). This implies that the occurring patterns do not affect connectivity, and therefore, activity and connectivity are in balance. Activity and connectivity are maintained because these networks are easily excitable and highly recurrent (le Feber et al. 2014), which leads to bursting firing patterns, with many action potentials within the time window of STDP.
Self-stabilizing activity is also obtained in artificial recurrent neural networks, and stable patterns are referred to as attractors (Amit and Brunel 1997). Although activity patterns in cultures stabilize after $3 \mathrm{wk}$, a slow drift of observed activity patterns (on timescales of hours to days) remains (Stegenga et al. 2008). A similar slow drift of self-sustained activity patters is observed in continuous attractor network models (Carter and Wang 2007).

Computer simulations robustly reproduced the experimentally observed stabilization of connectivity. To realize sufficient ongoing network activity and network bursts, the degree of connectivity (the relative number of connections per neuron) and the level of synaptic noise in the model were higher than biologically plausible. The relatively high degree ensured that all network models were highly recurrent and, combined with relatively high synaptic noise, produced bursting activity patterns. The current study confirms that isolated recurrent networks develop a stable connectivity. In biological networks, the development toward this stable connectivity is governed by the "rules" of STDP (le Feber et al. 2009), indicating that this process depends on synaptic plasticity.

An external input usually initiates deviating patterns, which may drive the network away from the existing balance. In vitro, we used a single stimulation electrode to directly (nonsynaptically) activate multiple neurons (Marom and Shahaf 2002; Wagenaar et al. 2004; Reinartz et al. 2014), and in simulations, we directly activated a set of neurons, which was usually sufficient to trigger a network burst. Stimuli were applied either as low-frequency pulses or as tetani. Tetanic stimulation has been shown to affect connectivity in a wide range of frequencies (20$250 \mathrm{~Hz})$, inter-train intervals $(2-10 \mathrm{sec})$, and numbers of pulses per train (10-100, see e.g., Jimbo et al. 1998, 1999; Ruaro et al. 2005). The exact parameter setting does not seem very critical,
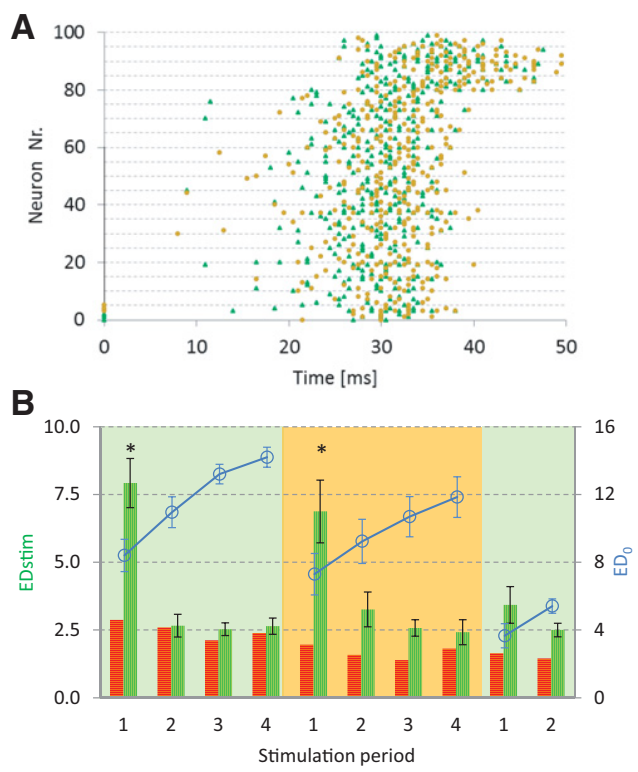

Figure 4. Response to two different stimuli in simulated network. $(A)$ example of stimulus response rasterplots (similarity: $\mathrm{EC}(\mathrm{A}[1], \mathrm{B}[1])=$ $0.95)$. Neurons $(0,1,2)(\operatorname{stim} A, \Delta)$, or $(3,4,5)(\operatorname{stim} B, \bullet)$ were stimulated at $t=0$. Neurons 80-99 were inhibitory. (B): Euclidean distances between connectivities across subsequent stimulation periods (vertically hatched bars). First, stimulus A was applied four times, then four times stimulus $B$ and then again stimulus $A$ ( $n=4$ simulations), horizontally hatched bars indicate distances between equidistant periods without stimulation $(n=1)$. Significant differences (ANOVA + Tukey's HSD, $P<$ 0.05 ) are indicated by an asterisk. 
as long as the amplitude is sufficient to induce a network response. This view is supported by the current finding that lowfrequency and tetanic stimulation yielded very similar results. Following a period of external stimulation, networks developed a new balance due to the mutually affective forces between activity and connectivity, with activity patterns including the stimulus response pattern. In simulations, adaptation of network connectivity was reflected by gradually changing stimulus responses during the early phase of the stimulation period toward a stabile response in the second half of the stimulation period. Furthermore, in all simulations the similarity between stimulus responses, particularly stabile stimulus responses, and post-stimulation bursts was significantly higher than that between stimulus responses and prestimulation bursts. This indicates that the stimulus response patterns appeared in the post-stimulation spontaneous activity. We saw a similar but non-significant trend in the experimental data. Indirect support that the new equilibrium includes the stimulus response patters, comes from the observation that subsequent application of the same input no longer affects connectivity. Although the connectivity changes across stimulation periods did not drop to zero, the remaining connectivity changes did not yield an increasing distance from the initial connectivity and therefore probably reflect fluctuations around a stable point. Apparently, the stimulus no longer formed a reorganizing force to drive connectivity further away from the initial state. The small values of $\mathrm{ED}$, in the order of $10^{-3}$, reflect the strengths of functional connections, which usually ranged from $10^{-4}$ to $10^{-2}$ (e.g., le Feber et al. 2007) and should therefore not be interpreted as minor connectivity changes. Investigation of artificial neural networks showed that external input usually changes the set of attractors in a network (Amit 1989). The changes in the connectivity matrix $S$ probably reflect such a change in the set of attractors, and the correspondingly adapted activity patterns can be interpreted as memory traces.

Nakazawa et al. (2004) defined four key features of a memory trace: (1) the memory trace should form in an experiencedependent manner, (2) the trace should be specific to the information that is acquired, (3) the trace must outlast the period during which the animal is exposed to the information, and (4) the subsequent presentation of at least part of the original cues should reactivate the trace. The connectivity changes in this study comply with conditions 1-3 and probably also with the fourth condition. Connectivity changes occur upon external stimulation, which can be seen as experience-dependent formation. Different inputs induce different connectivity changes and therefore the trace is specific to the acquired "information," and connectivity changes clearly outlasted the stimulation period. The current study focused on the formation of memory traces, and not on memory recollection. Therefore, compliance with the fourth criterion could only be deduced from indirect evidence. The finding that stimulus responses stabilized, probably means that subsequent presentation of the cue reactivated the trace and did not interfere with the established activity-connectivity equilibrium. Marom and coworkers showed that repeated stimulation of cultured cortical networks induced site-specific responses that enabled deduction of the stimulation site during up to $24 \mathrm{~h}$ (Shahaf et al. 2008; Kermany et al. 2010). This further supports the view that presentation of the original cue reactivates the trace.

From the waning effect of a repeated stimulus on connectivity, we can conclude that inputs are stored in network connectivity, but the encoding scheme remains unclear. Our findings imply that there is no univocal relationship between the stimulus and its encoding, as it depends not only on the stimulus but also on connectivity at the moment of arrival of the stimulus. Simulations showed that stimulus responses and spontaneous patterns developed toward each other, until the stimulus response no longer exerted a driving force away from the existing equilibrium. Spontaneous appearance of response patterns may be interpreted as a memory trace. Similar findings were obtained in vivo by Yao et al. (2007), who showed that response patterns to visual stimuli in cat visual cortex also appeared in spontaneous patterns. Their study, however, did not address network connectivity, and a mechanism to explain the appearance of the response patterns in the post-stimulation spontaneous activity patterns was not determined.

Stimulation at a second, different electrode, induced connectivity changes similar to the first stimulus. Again, large connectivity changes occurred across the first stimulation period, and much smaller changes upon subsequent periods. This pattern indicates that the network also memorized the second input. Moreover, it confirmed that the network was still able to adapt to external inputs, and that the unaffected connectivity after several repetitions of the first stimulus was not due to impeded network plasticity. This strongly supports the view that there was no longer a driving force away from the existing connectivity.

Returning to the first stimulus did not affect network connectivity, indicating that the first memory trace was not erased by application of the second stimulus. Instead, both memory traces existed in parallel, at least for the 4-h periods in our experiment. The longer-term characterization of memory traces remains subject to further study, but should be feasible with the current approach. We were able to detect memory traces after a few hours, whereas memory transfer from hippocampus to cortex probably takes at least several days (Wang et al. 2006; Rudy and Sutherland 2008). This accelerated formation of detectable traces may be related to model artifacts, like the relatively small network size. Alternatively, it may be easier to track memory traces through functional network connectivity than by extensively searching for certain patterns in recorded spontaneous activity. Thus, newly formed memory traces may be detected earlier. The simplicity and relatively long duration of our cues possibly further accelerated the formation of memory traces. In general, longer stimulation periods led to faster establishment of a new balance (data not shown), indicating that the duration of the stimulus may influence the time required before a memory trace can be detected.

The computational model, with three incorporated plasticity mechanisms: STF, STD, and STDP, perfectly reproduced these experimental findings. STD has been shown to destabilize attractors in artificial neural networks (Sandberg et al. 2003), but it is added to the computational model to avoid endless bursts of activity, in analogy to the presumed mechanism of burst termination in cultured networks (Eytan and Marom 2006). The implemented STDP enabled network connectivity to adapt to different inputs. However, occasionally stimulation leads to connectivity close to the borders of the connectivity space, with many synapses near their maximum strength or zero. In such cases, more extensive stimulation was required to pull the network out of this state toward another equilibrium. In simulations, tetanic stimulation was more suitable than low-frequency stimulation for retaining connectivity away from these borders. Network activation in the computer model tended to potentiate strong synapses and to depress weaker synapses. Second and subsequent pulses of a tetanus coinciding with an induced network burst, frequently forced synapses away from their extreme values, and kept networks in a state more susceptible to memorizing new inputs. Possibly, addition of a third, homeostatic plasticity mechanism that has been demonstrated in in vitro networks, synaptic scaling (Turrigiano et al. 1998), to the computer model could avoid these artifacts. Larger network models imposed a very high computational load and were not necessary to reproduce the experimental findings. However, it has been shown that the storage capacity of artificial neural networks, or the number of possible stable states 
(attractors), scales linearly with the number of synapses per neuron (Amit 1989) and, therefore, parallel storage of memory traces may even be better facilitated in larger networks.

We conclude that cultured cortical networks memorize external inputs. A second input did not erase the first memory trace, but both traces were stored in parallel. Three synaptic plasticity mechanisms, STDP, STF, and STD were sufficient to enable parallel memorization of different inputs, in networks without specific circuitry constraints.

\section{Materials and Methods}

\section{Cell culturing and stimulation}

We obtained cortical cells from newborn Wistar rats at postnatal Day 1. All surgical and experimental procedures complied with Dutch and European laws and guidelines and were approved by the Utrecht University Animal Experiments Review Committee. After trypsin treatment cells were dissociated by trituration. About 400,000 dissociated neurons ( $400 \mu \mathrm{L}$ suspension) were plated on a multielectrode array (MEA; Multi Channel Systems), precoated with polyethyleneimine. This procedure resulted in an initial cell density of $\sim 5000$ cells $/ \mathrm{mm}^{2}$, with aging cell densities gradually decreased to $\sim 2500$ cells $/ \mathrm{mm}^{2}$. Neurons were cultured in a circular chamber with inner diameter $d=20 \mathrm{~mm}$, glued on top of an MEA, which contained 60 titanium nitride electrodes with a $30-\mu \mathrm{m}$ diameter and $200-\mu \mathrm{m}$ pitch (Fig. 1A). The culture chamber was filled with $\sim 700 \mu \mathrm{L}$ R12 medium (Romijn et al. 1984). MEAs were stored in an incubator, under standard conditions of $37^{\circ} \mathrm{C}, 100 \%$ humidity, and $5 \% \mathrm{CO}_{2}$ in air. For recording, we firmly sealed the culture chambers with watertight but $\mathrm{O}_{2}$ and $\mathrm{CO}_{2}$ permeable foil (MCS; ALA scientific), and placed the cultures in a measurement setup outside the incubator. During recording, we kept the $\mathrm{CO}_{2}$ level of the environment $\sim 5 \%$ and we maintained humidity. For details about the recording setup see Stegenga et al. (2008). Recordings began after an accommodation period of at least $20 \mathrm{~min}$.

We used 20 different cultures for 21 experiments, which were performed $27 \pm 10 \mathrm{~d}$ after plating of the cells (see Table $1)$. Cultures were electrically stimulated with biphasic current pulses, of $200 \mu \mathrm{sec}$ per phase. Figure 5B shows an example of 5 min of recorded activity. Each tick after one of the electrodes 0-59 indicates an action potential recorded at that channel. The first $75 \mathrm{sec}$ of this recording contained low-frequency stimulation, indicated by ticks at channel 60 . Figure 5C shows examples of a stimulus response (left) and a spontaneous network burst (right).

All electrodes were probed at several amplitudes in random order, to find suitable electrodes for stimulation. There were no constraints related to the location of the stimulation electrodes. The amplitude was set to a level that usually ( $>50 \%$ of stimuli) triggered a network burst in response to a stimulus pulse at the selected electrode (12-24 $\mu \mathrm{A})$. At these currents, voltages remained sufficiently low to avoid electrolysis. Pulses were applied at a single electrode, either as low-frequency pulses for $10 \mathrm{~min}$ (interpulse interval: $3-5 \mathrm{sec}$ ), or as tetani (trains of 10 pulses at 100 $\mathrm{Hz}$, inter-train interval: $5 \mathrm{sec}$, duration: 10-15 min). Subsequent stimulation periods were separated by $1 \mathrm{~h}$ of no stimulation. Periods of no stimulation were used to record spontaneous activity, needed to infer functional connectivity. In low-frequency stimulation experiments, we calculated the area under the PSTH to quantify the network stimulus response. In tetanus experiments, this was not possible because the mean stimulus response time window largely exceeded the inter-pulse interval. In tetanus experiments, we monitored the array wide activity level during periods of no stimulation to verify that the cultures did not deteriorate during the experiments.

First experiments showed quickly decreasing connectivity changes in response to repeated periods of stimulation at a certain electrode. After 3-4 stimulation periods at a certain electrode only minor connectivity changes were found, not or hardly exceeding spontaneous fluctuations (see Fig. 1B). In experiments with sepa-

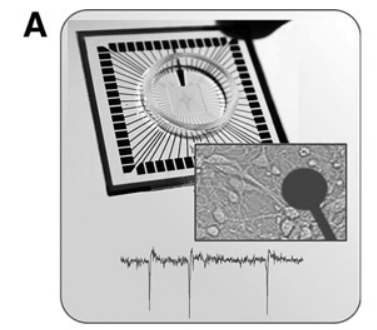

C

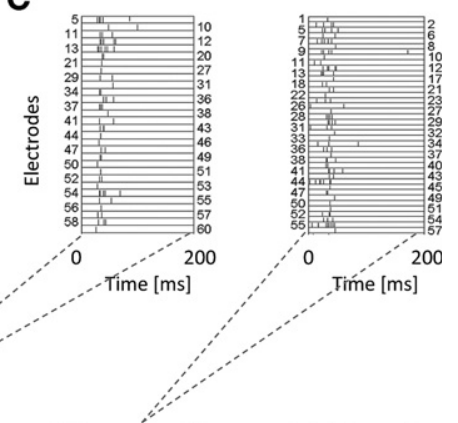

B

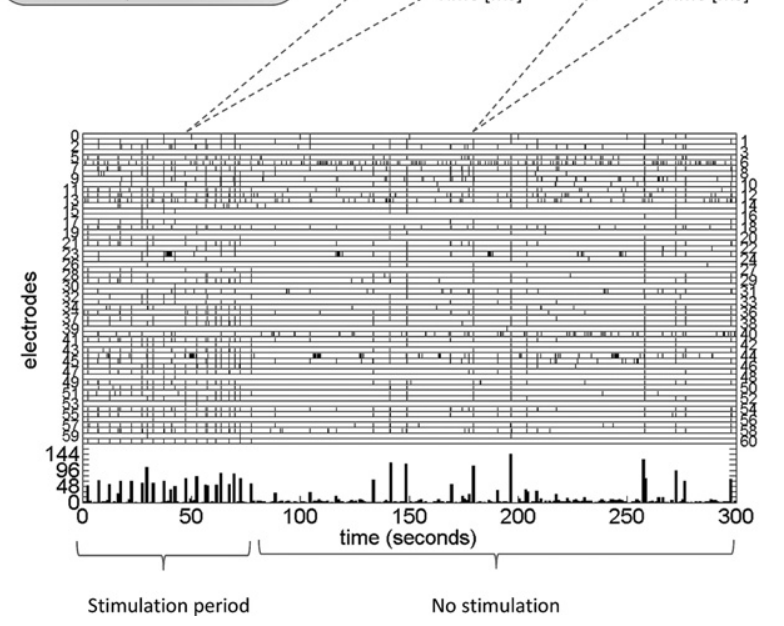

Figure 5. Multielectrode array (MEA) and recorded activity. $(A)$ MEA, zoom at one of the electrodes and several neurons, and an example of extracellularly recorded action potentials. (B) Raster plot of recorded activity. Vertical axes indicate all electrodes, action potentials are shown as ticks in the row corresponding to the recording electrode. Ticks in row 60 indicate stimulus pulses. (C) Examples of a stimulus response (left) and a spontaneous network burst (right).

rate periods of stimulation at different electrodes, we aimed to reach this "saturation point" for each electrode, while keeping the total experimental time as short as possible to avoid spontaneous connectivity changes, preferably $<15 \mathrm{~h}$ (Stegenga et al. 2008). Multiple stimulation site experiments contained four stimulation periods at one electrode, then four periods of stimulation at a second electrode and finally four stimulation periods at the first electrode, and lasted $\sim 12-13 \mathrm{~h}$.

\section{Connectivity analysis}

We used periods of spontaneous activity, including population bursts as well as periods of more dispersed firing, to analyze network connectivity. Long-term recordings were divided into data blocks of $2^{13}$ spiking events. The 1-h epochs between subsequent stimulation periods were long enough to obtain multiple data blocks in all experiments, and short enough to keep the total experimental time below $15 \mathrm{~h}$. In each data block, we used conditional firing probabilities (CFPs) to determine functional connectivity (le Feber et al. 2007). For all possible pairs of active electrodes ( $>250$ spikes/data block) we calculated CFPs as the probability to record an action potential at electrode $j$ at $t=\tau(\tau>0)$, given that one was recorded at electrode $i$ at $t=0$. If a CFP curve was not flat (for exact criteria see le Feber et al. (2007), the two neurons were functionally connected. Functional connections were characterized by two parameters: their strength and latency. These parameters may be used to follow the development of a functional connection in time.

In each data block, the strengths of all connections were combined into a connectivity matrix $\mathrm{S}$, where $\mathrm{S}(i, j)$ contains the strength of the functional connection from $i$ to $j$. The magnitude of changes between subsequent data blocks was assessed by the Euclidean distance (ED) between connectivity matrices at time $t$ 
and time $t_{0}$ (Equation 1).

$$
\mathrm{ED}_{t_{0}}(t)=\sqrt{\sum_{i=1}^{n} \sum_{j=1}^{n}\left[S_{i j}(t)-S_{i j}\left(t_{0}\right)\right]^{2}}
$$

$t_{0}$ was always smaller than $t$ and chosen as:

The first data block of a recording, or in the case of stimulation (possibly at multiple electrodes), the last data block before stimulation at that specific electrode. These distances were indicated by $\mathrm{ED}_{0}$; or

the last data block that was separated from $t$ by a stimulation period. These distances were indicated by $\mathrm{ED}_{\text {stim. }}$.

The total number of functional connections widely differed between cultures, and consequently, absolute ED values covered a wide range. Therefore, we normalized all ED curves per culture to their mean value before statistical testing.

\section{Activity pattern analysis}

During phases of no stimulation, network bursts were extracted using the algorithm by Eckmann et al. (2008) and categorized as pre- and post-stimulus bursts. To assess the similarity between stimulus responses and spontaneous bursts, we adopted a method by Segev et al. (2004), which was adapted to yield normalized values between 0 and 1 . In short, at all electrodes signals were represented as point processes and convoluted with a normalized Gaussian. We calculated cross correlations per electrode, and used the maximum mean cross correlation (at one certain latency) as a measure of similarity (events correlation; EC).

\section{Computer modeling}

We used a 100-neuron computer model to verify that (1) without external input networks established a stable connectivity, (2) external input disrupted this equilibrium, (3) networks reached a new stable connectivity, and (4) this new equilibrium was no longer disrupted by external input.

To simulate the firing activity, we used a (pulse-coupled) spiking neural network model as described in Gritsun et al. (2010, 2011). We used a set of neuronal parameters that adequately reproduced the dynamics of cortical neurons (Izhikevich 2003), for details see "Neuron model." This set contained a mixture of all neuronal cell types that exist in the cortex, which gave our simulations a certain degree of robustness against variations of cell properties. However, it should be noted that our results might be affected by changes in cell properties. On average, each neuron had 50 connections. This implicates that the probability that any pair of two neurons were monosynaptically connected was 50\%. This very high connection probability was necessary to enable the neurons to trigger each other, and ensured a highly recurrent network. The connections were modeled by synapses that expressed STF and STD as described by Markram et al. (1998). In addition to the model by Gritsun et al., synapses also expressed additive (Song et al. 2000) or multiplicative STDP (Van Rossum et al. 2000), implemented with a symmetric implementation of a nearest neighbor pairing scheme. We applied parameter values obtained by Gupta et al. (2000) and Markram et al. (1998) to describe the phenomenological synaptic model of short-term plasticity, including STD and STF, for details on the synapse model see "Synapse model." Ongoing spontaneous activity was initiated by white synaptic noise as implemented by Gritsun et al. (2012), see "Synaptic noise and model sensitivity." Synaptic noise was set to a level high enough to ensure that all models displayed spontaneous activity, including network bursts. This indicated that neurons were not solely driven by noise input, but were able to trigger each other. Connectivity matrices containing the strengths of all synapses were stored every minute to observe the development of connectivity under various conditions of stimulation or no stimulation. To validate the solidity of our findings, we repeated all experiments in multiple simulations, based on different network realizations (different set of neurons and a different connectivity matrix), different noise realizations (with the same stochastic characterization), and different STDP models (see "Synaptic noise and model sensitivity"). A more thorough sensitivity analysis of this type of models can be found in Gritsun et al. (2010, 2011, 2012). The model was implemented in Matlab and $\mathrm{C}++$, codes are available upon request.

We aimed to start stimulation only in networks with stabilized connectivity. To quantify the stability of connectivity, we calculated $\mathrm{ED}_{0}$ for all connectivity matrices. Connectivity stabilized as the slope of the $\mathrm{ED}_{0}$ curve against time approached zero. Connectivity was considered stable when this slope dropped below $1 \%$ of the slope based on the first five data points. Before computer models were stimulated, we ensured that sufficient time was allowed to develop a stable connectivity. This stable connectivity was used as the reference to calculate $\mathrm{ED}_{0}$.

To simulate electrical stimulation, we simultaneously activated a random set of three (all excitatory) or nine neurons (two inhibitory and seven excitatory), which was in general sufficient to trigger a network response. This approach is conceptually congruent with experimental stimulation, where even stimulation through a single electrode activates a set of nearby neurons and neurons that have an axon passing close to the stimulation electrode, which together may trigger a reverberating network response. Stimulation was applied as either low-frequency stimuli $(0.2 \mathrm{~Hz}$, for $15 \mathrm{~min}$ at 9 neurons, see Fig. $3 \mathrm{~A}$, or $0.125 \mathrm{~Hz}$ for $2 \mathrm{~h}$ at 3 neurons, see Fig. 3B) or tetani, as described under cell culturing and stimulation. To calculate $\mathrm{ED}_{\text {stim }}$ in simulations, we used the mean weights of all synapses during the last $15 \mathrm{~min}$ before stimulation and the first 15 min after stimulation. $\mathrm{ED}_{\text {stim }}$ values that significantly exceeded those of connectivity matrices without stimulation in between were indicative of a disturbed balance, whereas $\mathrm{ED}_{\text {stim }}$ values in the range of spontaneous fluctuations confirmed that the stimulus was compatible with the connectivity.

\section{Neuron model}

The structure of the network was based on the network model by Gritsun et al. (2010). Eighty percent of the neurons in the model were excitatory and 20\% were inhibitory. Each neuron $i$ spiked when the intracellular potential $f_{i}$ reached the threshold of $30 \mathrm{mV}$ :

$$
x_{i}(t)= \begin{cases}1 & \text { if } f_{i} \geq 30 \mathrm{mV} \\ 0 & \text { otherwise }\end{cases}
$$

Each time step the postsynaptic potentials $\left(\mathrm{psp}_{i j}\right)$ of all presynaptic neurons were added to the membrane potential $v_{i}$ :

$$
f_{i}\left(v_{i}, \operatorname{psp}_{i j}\right)=v_{i}(t)+\sum_{j=1}^{n} \operatorname{psp}_{i j}
$$

with $n$ the number of neurons.

The membrane potential was described using two differential equations as described in Izhikevich (2003):

$$
\begin{gathered}
\frac{\mathrm{d} v_{i}}{\mathrm{~d} t}=0.04 v_{i}^{2}+5 v_{i}+140-u_{i}+I \\
\frac{\mathrm{d} u_{i}}{\mathrm{~d} t}=a_{i}\left(b_{i} v_{i}-u_{i}\right)
\end{gathered}
$$

with a resetting of the variables $v$ and $u$ after spiking:

$$
\text { if } v_{i} \geq 30 \mathrm{mV}, \quad \text { then }\left\{\begin{array}{c}
v_{i} \leftarrow c_{i} \\
u_{i} \leftarrow u_{i}+d_{i}
\end{array}\right.
$$

Here, $v_{\mathrm{i}}$ and $u_{i}$ are the membrane potential and the recovery current. The recovery current represents the activation of $\mathrm{K}^{+}$and the inactivation of $\mathrm{Na}^{+}$currents. The time scaling of recovery was described by $a_{i}$, with lower $a_{i}$ resulting in slower recovery. $b_{i}$ described the sensitivity of $u_{\mathrm{i}}$ for subthreshold variations of $v_{\mathrm{i}} c_{i}$ 
and $d_{i}$ describe the after-spike reset value of $v_{i}$ and $u_{\mathrm{i}} \cdot c_{i}$ represents the fast high-threshold $\mathrm{K}^{+}$conductance and $d_{i}$ the slow highthreshold $\mathrm{K}^{+}$and $\mathrm{Na}^{+}$conductances. I was the input to the neuron with arbitrary units and can be used for synaptic currents or injected DC currents. All models contained a mixture of the different inhibitory (fast spiking and low-threshold-spiking neurons) and excitatory neuron types (regular spiking, intrinsically bursting, and chattering neurons). The mixture was created by assigning the following values to the parameters for excitatory neurons:

$$
\begin{aligned}
& \left(a_{i}, b_{i}\right)=(0.02,0.2) \\
& \left(c_{i}, d_{i}\right)=(-65,8)+(15,-6) r_{i}{ }^{2}
\end{aligned}
$$

and to the inhibitory neurons:

$$
\begin{aligned}
& \left(a_{i}, b_{i}\right)=(0.02,0.25)+(0.08,-0.05) r_{i} \\
& \left(c_{i}, d_{i}\right)=(-65,2)
\end{aligned}
$$

in which $r_{i}$ is a random variable uniformly distributed over [0;1]. In Equation (7) $r_{i}$ was squared to create a bias toward RS neurons (Izhikevich 2004).

\section{Synapse model}

Upon the arrival of new synaptic input the postsynaptic potential was increased (excitatory input) or decreased (inhibitory), the amplitude of this change reflected the synaptic efficacy $\left(\mathrm{se}_{i j}\right)$. Otherwise, the PSP declined to baseline with time constant $\tau_{\text {syn }}$. We took $\tau_{\text {syn }}=5 \mathrm{msec}$ for both excitatory and inhibitory neurons, based on the time constants of the AMPA and the GABA synaptic conductances (5.3 and $5.6 \mathrm{msec}$, respectively (Dayan and Abbott 2000)).

$$
\frac{\mathrm{dpsp}_{i j}}{\mathrm{~d} t}=-\frac{\mathrm{psp}_{i j}}{\tau_{\mathrm{syn}}}+\mathrm{se}_{i j} \mathrm{e}_{i j} \delta\left(t-t_{j}^{\mathrm{sp}}-l_{i j}\right)
$$

where, $t_{j}^{\mathrm{sp}}$ is the time of spiking of the postsynaptic neuron, $e_{\mathrm{ij}}$ is 1 when the presynaptic neuron $(j)$ was excitatory, -1 when this neuron was inhibitory, and 0 if there was no connection between the neurons.

The latency $\left(l_{\mathrm{ij}}\right)$ is defined as the total delay between generation of the action potential in neuron $j$ and arrival of the postsynaptic potential in neuron $i$. $i_{i j}$ is normally distributed between 3.5 and the maximal latency $\left(D_{\max }\right) . D_{\max }$ is estimated to be $25 \mathrm{msec}$ in cortical cultures (Gritsun et al. 2010). We maintained this value of $D_{\max }$, even in the current, much smaller network model to avoid that a small $D_{\max }$ of a few milliseconds, combined with the time step of $1 \mathrm{msec}$ would give all neuron pairs approximately the same latency.

The synaptic efficacy represented different processes of synaptic plasticity: $\mathrm{se}_{i j}=F \cdot a_{i j} \cdot r_{i j} \cdot y_{i j} \cdot w_{i j}$, in which $r_{i j}, y_{i j}$, and $w_{i j}$ are variables giving the relative strength of the synapse. $r_{i j}$ is relat-

\begin{tabular}{|c|c|c|}
\hline Variable & Initial value & Comment \\
\hline $\begin{array}{l}v_{\mathbf{i}} \\
f_{i}\end{array}$ & $-65 \mathrm{mV}$ & \\
\hline$f_{\mathbf{i}}$ & $-65 \mathrm{mV}$ & \\
\hline$u_{i}$ & $b_{\mathrm{i}} \cdot v_{\mathrm{i}}$ & Dependent on neuron type \\
\hline$r_{i j}$ & 1 & \\
\hline$y_{i j}$ & & Dependent on synapse type \\
\hline$w_{i j}$ & Between 0 and 1 & $\begin{array}{l}\text { Dependent on synapse type, } \\
\text { initially uniformly distributed }\end{array}$ \\
\hline
\end{tabular}
ed to the fraction of neurotransmitter available and represents short-term depression, $y_{i j}$ represents STF, and $w_{\mathrm{ij}}$ is a factor determined by STDP. Initial values are shown in Table $2 . F$ and $a_{i j}$ are

Table 2. Initial values of the variables

$\left(f_{i}\right)$ intracellular potential, $\left(v_{i}\right)$ membrane potential, $\left(u_{i}\right)$ recovery current. $r_{i j}$ represents short-term depression, $y_{i j}$ represents STF, and $w_{i j}$ is a factor determined by STDP. parameters that give the baseline strength of the synapse. The value of $a_{i j}$ depends on the type of synapse as shown in Table 3 . With these values of $a_{i j}$, the value of se corresponds to biologically plausible EPSP values if $F=1 . F$, a compensation factor to achieve bursting patterns in the small network with few inputs per neuron was equal for all synapses and was set to a value necessary for bursting.

\section{Short-term plasticity}

STF and depression were implemented using the model of Markram et al. (1998).

With presynaptic spikes, $r$ and $y$ were updated according to the following equations:

$$
\begin{gathered}
r_{i j}^{k}=r_{i j}^{k-1}\left(1-y_{i j}^{k}\right) \mathrm{e}^{-\Delta t_{\text {STP }} / \tau_{\text {rec }}}+1-\mathrm{e}^{-\Delta t_{\text {STP }} / \tau_{\text {rec }}} \\
y_{i j}^{k}=y_{i j}^{k-1} \mathrm{e}^{-\Delta t_{\text {STP }} / \tau_{\text {facil }}}+u_{i j}\left(1-y_{i j}^{k-1} \mathrm{e}^{-\Delta t_{\text {STP }} / \tau_{\text {facil }}}\right)
\end{gathered}
$$

In these equations, $\Delta t_{\mathrm{STP}}$ is the time difference between the previous and the current presynaptic spike, $u_{i j}$ is the release probability for the first spike and $\tau_{\text {rec }}$ and $\tau_{\text {facil }}$ are time constants for the recovery and facilitation, respectively. $k$ is the running variable for the spikes. For instance, $r_{i j}^{\mathrm{k}}$ refers to the fraction of neurotransmitter available with the current spike in the synapse between neuron $j$ and $i$. Table 3 shows the values for $u_{i j}, \tau_{\text {rec }}$, and $\tau_{\text {facil }}$.

\section{Spike-timing-dependent plasticity}

Both the additive and the multiplicative STDP model update $w$ at every pre- and postsynaptic spike: $w_{i j}^{k+1}=w_{i j}^{k}+\Delta w$. STDP was only implemented in excitatory connections; if the presynaptic neuron was inhibitory, $w$ was constant and equal to 0.5 . Both models were based on measurements of EPSC. This means that they actually model how the EPSC instead of the EPSP is influenced by STDP. However, it was assumed that these were linearly correlated; so these equations could also be used to model the influence of STDP on the EPSP.

The additive STDP model (Song et al. 2000) used the following update rules:

$$
\Delta w_{i j}= \begin{cases}A_{-} \mathrm{e}^{\Delta t_{\mathrm{STDP}} / \tau_{-}} & \text {if } \Delta t_{\mathrm{STDP}}<0 \\ A_{+} \mathrm{e}^{-\Delta t_{\mathrm{STP}} / \tau_{+}} & \text {if } \Delta t_{\mathrm{STDP}} \geq 0\end{cases}
$$

with $A_{+}=0.005, A_{-}=-1.05 A_{+}$, and $\tau_{-}=\tau_{+}=20 \mathrm{msec} . \Delta t_{\mathrm{STDP}}$ is the time difference between the presynaptic and the postsynaptic spike, $\Delta t_{\text {STDP }}=t_{\text {post }}-\left(t_{\text {pre }}+l_{i j}\right)$. Furthermore, we limited $w_{i j}$ to $0 \leq$ $w_{i j} \leq 1$ to avoid instability.

The value of $A_{-}$followed from the restriction that the product of the probability of potentiation and amount of potentiation should equal the product of the probability of depression and amount of depression. The probability of a postsynaptic spike just after a presynaptic spike is higher than a presynaptic spike just after a postsynaptic spike, because presynaptic spike can

Table 3. Values of the parameters used in the synapse model for different synapse types

\begin{tabular}{llccc}
\hline & $\boldsymbol{u}_{\text {ij }}$ & $\tau_{\text {rec }}(\mathrm{ms})$ & $\tau_{\text {facil }}(\mathrm{ms})$ & $\boldsymbol{a}_{\mathrm{ij}}(\mathrm{mV})$ \\
\hline $\mathrm{EE}$ & 0.59 & 813 & 0 & 1.8 \\
$\mathrm{EI}$ & 0.049 & 399 & 1797 & 5.4 \\
$\mathrm{IE}$ & 0.16 & 45 & 376 & 7.2 \\
$\mathrm{II}$ & 0.25 & 706 & 21 & 7.2 \\
\hline
\end{tabular}

EE is a synapse between two excitatory neurons, El between an excitatory (presynaptic) and inhibitory (postsynaptic), IE between inhibitory and excitatory and II between two inhibitory neurons. $a_{i j}$ gives the baseline strength of the synapse, $u_{i j}$ is the release probability for the first spike and $\tau_{\text {rec }}$ and $\tau_{\text {facil }}$ are time constants for the recovery and facilitation. The values of $u_{i j}, \tau_{\text {rec }}$ and $\tau_{\text {facil }}$ are taken from Gritsun et al. (2010), the values of $a_{i j}$ from Tsodyks et al. (2000). 
initiate postsynaptic spike, but not vice versa. Thus, the mean amount of depression should be lower than the mean amount of potentiation (Song et al. 2000).

The multiplicative model (Van Rossum et al. 2000) was almost equal to the additive model, except that the weight update of the depression depended on the weight. The same parameter values were used, but the value of $A_{-}$was adapted to depend on the current weight. model:

This gave the following update rules for the multiplicative

$$
\Delta w_{i j}= \begin{cases}A_{-} w_{i j}{ }^{\Delta t_{\mathrm{STDP}} / \tau_{-}} & \text {if } \Delta t_{\mathrm{STDP}}<0 \\ A_{+} \mathrm{e}^{-\Delta t_{\mathrm{STDP}} / \tau_{+}} & \text {if } \Delta t_{\mathrm{STDP}} \geq 0\end{cases}
$$

Here, we took $A_{-}=-0.0105$, assuming that $w_{i j}$ averaged $\sim 0.5$. In this, STDP model boundaries were not necessary.

\section{Synaptic noise and model sensitivity}

Synaptic noise initiated network spiking and bursting behavior. Synaptic noise was injected as white noise added to the synaptic current $I$ (see Equation 4). This approach avoided an undesired influence of the time step size on the solution of the differential equations.

The mean value of the synaptic noise $(\mu)$ was 0 . Simulations with different values of the compensation factor of the synaptic strength $(F)$, the standard deviation of the noise $(\sigma)$, and the maximal latency $\left(D_{\max }\right)$ were done to assess the influence of these parameters on the number of bursts, the duration of these bursts and the spike frequency during these bursts. This was done with the additive as well as the multiplicative STDP model. The simulation time was $5 \mathrm{~min}$ and the initial weights of the synapses $(w)$ were uniformly distributed between 0 and 1 . The neuron parameters $(a, b, c$, and $d)$ and the connectivity matrix $(L)$ were equal in all simulations, except for the simulations in which $D_{\max }$ was varied, when all latencies increased or decreased proportionally to $D_{\max }$. Simulations with the following parameter values were performed:

- $F$ ranged between 4 and 10 with steps of 1 . These simulations were done with $\sigma=2.0$ or $\sigma=2.5$. $D_{\max }$ was $25 \mathrm{msec}$.

- $\sigma$ Varied between 2.0 and 3.0 with steps of 0.1. $F$ was 5 and $D_{\max }$ was 25 msec.

- $D_{\max }$ was $3.5,5,10,15,20$, and 25 msec. In all these simulations, $F=5$ and $\sigma=2.1$.

\section{Acknowledgments}

The authors declare no competing financial interests. The authors thank Prof. Dr. M.J.A.M. van Putten, Prof. Dr. R.J.A. van Wezel, and Dr. F. Battaglia for fruitful discussions and their invaluable suggestions and comments on the manuscript, and Dr. G. Hassink for preparation of the cultures.

Author contributions: J.1.F. designed and supervised the study, and prepared the manuscript, T.W. and J.D. performed the in vitro experiments and, T.M.v.V. and J.D. wrote the computer model and performed the simulations. All authors contributed to the manuscript.

\section{References}

Amit DJ. 1989. Modeling brain function, the world of attractor networks. Cambridge University Press, Cambridge, UK.

Amit DJ, Brunel N. 1997. Model of global spontaneous activity and local structured activity during delay periods in the cerebral cortex. Cereb Cortex 7: 237-252.

Bramham CR, Messaoudi E. 2005. BDNF function in adult synaptic plasticity: the synaptic consolidation hypothesis. Prog Neurobiol 76: 99-125.

Carter E, Wang X-J. 2007. Cannabinoid-mediated disinhibition and working memory: dynamical interplay of multiple feedback mechanisms in a continuous attractor model of prefrontal cortex. Cereb Cortex 17: i16-i26.

Dayan P, Abbott LF. 2000. Theoretical neuroscience. MIT Press, Boston.

Eckmann JP, Jacobi S, Marom S, Moses E, Zbinden C. 2008. Leader neurons in population bursts of $2 \mathrm{~d}$ living neural networks. New J Phys $\mathbf{1 0 .}$

Eytan D, Marom S. 2006. Dynamics and effective topology underlying synchronization in networks of cortical neurons. J Neurosci 26: 8465-8476.

Frankland PW, Bontempi B. 2005. The organization of recent and remote memories. Nat Rev Neurosci 6: 119-130.

Gais S, Born J. 2004. Declarative memory consolidation: mechanisms acting during human sleep. Learn Mem 11: 679-685.

Gerard RW. 1949. Psychology and psychiatry. Am J Psychiatry 106: $161-173$.

Gritsun TA, le Feber J, Stegenga J, Rutten WL. 2010. Network bursts in cortical cultures are best simulated using pacemaker neurons and adaptive synapses. Biol Cybern 102: 293-310.

Gritsun T, le Feber J, Stegenga J, Rutten WL. 2011. Experimental analysis and computational modeling of inter-burst intervals in spontaneous activity of cortical neuronal cultures. Biol Cybern 105: 197-210.

Gritsun TA, le Feber J, Rutten WLC. 2012. Growth dynamics explain the development of spatiotemporal burst activity of young cultured neuronal networks in detail. PLoS One 7: e43352.

Gupta A, Wang Y, Markram H. 2000. Organizing principles for a diversity of GABAergic interneurons and synapses in the neocortex. Science 287: $273-278$.

Hebb DO. 1949. The organization of behavior. Wiley, New York.

Izhikevich EM. 2003. Simple model of spiking neurons. IEEE Trans Neural Netw 14: 1569-1572.

Izhikevich EM. 2004. Which model to use for cortical spiking neurons? IEEE Trans Neural Netw 15: 1063-1070.

Jimbo Y, Robinson HP, Kawana A. 1998. Strengthening of synchronized activity by tetanic stimulation in cortical cultures: application of planar electrode arrays. IEEE Trans Biomed Eng 45: 1297-1304.

Jimbo Y, Tateno T, Robinson HP. 1999. Simultaneous induction of pathway-specific potentiation and depression in networks of cortical neurons. Biophys J 76: 670-678.

Karlsson MP, Frank LM. 2009. Awake replay of remote experiences in the hippocampus. Nat Neurosci 12: 913-918.

Kermany E, Gal A, Lyakhov V, Meir R, Marom S, Eytan D. 2010. Tradeoffs and constraints on neural representation in networks of cortical neurons. J Neurosci 30: 9588-9596.

le Feber J, Rutten WL, Stegenga J, Wolters PS, Ramakers GJ, van Pelt J. 2007. Conditional firing probabilities in cultured neuronal networks: a stable underlying structure in widely varying spontaneous activity patterns. $J$ Neural Eng 4: 54-67.

le Feber J, Van Pelt J, Rutten WL. 2009. Latency-related development of functional connections in cultured cortical networks. Biophys J 96: $3443-3450$.

le Feber J, Stoyanova II, Chiappalone M. 2014. Connectivity, excitability and activity patterns in neuronal networks. Phys Biol 11: 036005.

Markram H, Wang Y, Tsodyks M. 1998. Differential signaling via the same axon of neocortical pyramidal neurons. Proc Natl Acad Sci 95: 5323-5328.

Marom S, Shahaf G. 2002. Development, learning and memory in large random networks of cortical neurons: lessons beyond anatomy. Q Rev Biophys 35: 63-87.

Nadel L, Winocur G, Ryan L, Moscivitch M. 2007. Systems consolidation and hippocampus: two views. Debates Neurosci 1: 55-66.

Nakashiba T, Buhl DL, McHugh TJ, Tonegawa S. 2009. Hippocampal CA3 output is crucial for ripple-associated reactivation and consolidation of memory. Neuron 62: 781-787.

Nakazawa K, McHugh TJ, Wilson MA, Tonegawa S. 2004. NMDA receptors, place cells and hippocampal spatial memory. Nat Rev Neurosci 5: 361-372.

Reinartz S, Biro I, Gal A, Giugliano M, Marom S. 2014. Synaptic dynamics contribute to long-term single neuron response fluctuations. Front Neural Circuits 8: 71

Romijn HJ, van Huizen F, Wolters PS. 1984. Towards an improved serum-free, chemically defined medium for long-term culturing of cerebral cortex tissue. Neurosc Biobehav Rev 8: 301-334.

Ruaro ME, Bonifazi P, Torre V. 2005. Towards the neurocomputer: image processing and pattern recognition with neuronal cultures. IEEE Trans Biomed Eng 52: 371-383.

Rudy JW, Sutherland RJ. 2008. Is it systems or cellular consolidation? Time will tell. An alternative interpretation of the Morris group's recent science paper. Neurobiol Learn Mem 89: 366-369.

Sandberg A, Tegnér J, Lansner A. 2003. A working memory model based on fast Hebbian learning. Network 14: 789-802.

Segev R, Baruchi I, Hulata E, Ben-Jacob E. 2004. Hidden neuronal correlations in cultured networks. Phys Rev Lett 92: 118102. 
Shahaf G, Eytan D, Gal A, Kermany E, Lyakhov V, Zrenner C, Marom S. 2008. Order-based representation in random networks of cortical neurons. PLoS Comput Biol 4: e1000228.

Song S, Miller KD, Abott LF. 2000. Competitive Hebbian learning through spike-timing-dependent synaptic plasticity. Nat Neurosci 3: 919-926.

Squire LR, Cohen NJ, Nadel L. 1984. The medial temporal region and memory consolidation: a new hypothesis. In Memory consolidation (ed. Weingartner H, Parker E), pp. 185-210. Lawrence Erlbaum, Hillsdale, NJ.

Stegenga J, le Feber J, Marani E, Rutten WL. 2008. Analysis of cultured neuronal networks using intraburst firing characteristics. IEEE Trans Biomed Eng 55: 1382-1390.

Sutherland GR, McNaughton B. 2000. Memory trace reactivation in hippocampal and neocortical neuronal ensembles. Curr Opin Neurobiol 10: $180-186$.

Tsodyks M, Uziel A, Markram H. 2000. Synchrony generation in recurrent networks with frequency-dependent synapses. J Neurosci 20: RC50.
Turrigiano GG, Leslie KR, Desai NS, Rutherford LC, Nelson SB. 1998. Activity-dependent scaling of quantal amplitude in neocortical neurons. Nature 391: 892-896.

van Pelt J, Wolters PS, Corner MA, Rutten WL, Ramakers GJ. 2004. Long-term characterization of firing dynamics of spontaneous bursts in cultured neural networks. IEEE Trans Biomed Eng 51: 2051-2062.

van Rossum MCW, Bi GQ, Turrigiano GG. 2000. Stable hebbian learning from spike timing-dependent plasticity. J Neurosci 20: 8812-8821.

Wagenaar DA, Pine J, Potter SM. 2004. Effective parameters for stimulation of dissociated cultures using multi-electrode arrays. J Neurosci Methods 138: $27-37$.

Wang H, Hu Y, Tsien JZ. 2006. Molecular and systems mechanisms of memory consolidation and storage. Prog Neurobiol 79: 123-135.

Yao H, Shi L, Han F, Gao H, Dan Y. 2007. Rapid learning in cortical coding of visual scenes. Nat Neurosci 10: $772-778$.

Received June 17, 2015; accepted in revised form September 8, 2015. 


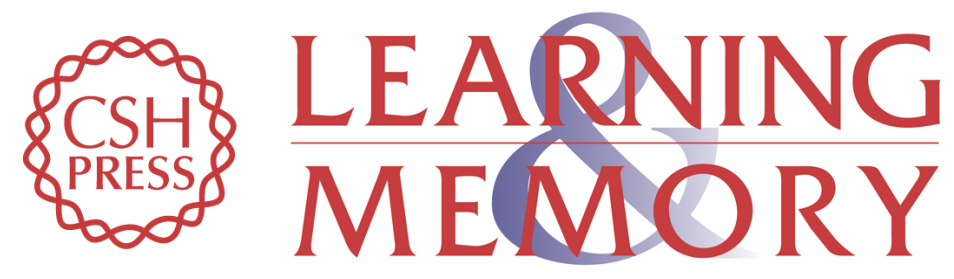

\section{Repeated stimulation of cultured networks of rat cortical neurons induces parallel memory traces}

Joost le Feber, Tim Witteveen, Tamar M. van Veenendaal, et al.

Learn. Mem. 2015, 22:

Access the most recent version at doi:10.1101/lm.039362.115

References This article cites 42 articles, 7 of which can be accessed free at:

http://learnmem.cshlp.org/content/22/12/594.full.html\#ref-list-1

Creative This article is distributed exclusively by Cold Spring Harbor Laboratory Press for the

Commons

first 12 months after the full-issue publication date (see

License http://learnmem.cshlp.org/site/misc/terms.xhtml). After 12 months, it is available under a Creative Commons License (Attribution-NonCommercial 4.0 International), as described at http://creativecommons.org/licenses/by-nc/4.0/.

Email Alerting Receive free email alerts when new articles cite this article - sign up in the box at the Service top right corner of the article or click here. 